\title{
Sharp Inequalities Involving Neuman Means of the Second Kind with Applications
}

\author{
Lin-Chang Shen ${ }^{1}$, Yue-Ying Yang ${ }^{2 *}$ and Wei-Mao Qian ${ }^{3}$ \\ ${ }^{1}$ Huzhou Shanlian Adult School,Huzhou, Zhejiang,China \\ ${ }^{2}$ Mechanic Electronic and Automobile Egineering College, Huzhou Vocational \& Technical College, \\ Huzhou, Zhejiang,China \\ ${ }^{3}$ School of Distance Education, Huzhou Broadcast and TV University, Huzhou, Zhejiang, China \\ Email: yyy1008hz@163.com.
}

\begin{abstract}
In this paper, we give the explicit formulas for Neuman means of the second kind $N_{G Q}(a, b)$ and $N_{Q G}(a, b)$, and find the best possible parameters $\alpha_{i}, \beta_{i} \in(0,1)(i=1,2,3, \cdots, 6)$ such that the double inequalities

$$
\begin{aligned}
& \alpha_{1} Q(a, b)+\left(1-\alpha_{1}\right) G(a, b)<N_{Q G}(a, b)<\beta_{1} Q(a, b)+\left(1-\beta_{1}\right) G(a, b), \\
& \frac{\alpha_{2}}{G(a, b)}+\frac{1-\alpha_{2}}{Q(a, b)}<\frac{1}{N_{Q G}(a, b)}<\frac{\beta_{2}}{G(a, b)}+\frac{1-\beta_{2}}{Q(a, b)}, \\
& \alpha_{3} Q(a, b)+\left(1-\alpha_{3}\right) G(a, b)<N_{G Q}(a, b)<\beta_{3} Q(a, b)+\left(1-\beta_{3}\right) G(a, b), \\
& \frac{\alpha_{4}}{G(a, b)}+\frac{1-\alpha_{4}}{Q(a, b)}<\frac{1}{N_{G Q}(a, b)}<\frac{\beta_{4}}{G(a, b)}+\frac{1-\beta_{4}}{Q(a, b)}, \\
& \alpha_{5} Q(a, b)+\left(1-\alpha_{5}\right) V(a, b)<N_{Q G}(a, b)<\beta_{5} Q(a, b)+\left(1-\beta_{5}\right) V(a, b), \\
& \alpha_{6} Q(a, b)+\left(1-\alpha_{6}\right) U(a, b)<N_{G Q}(a, b)<\beta_{6} Q(a, b)+\left(1-\beta_{6}\right) U(a, b),
\end{aligned}
$$
\end{abstract}

holds for all $a, b>0$ with $a \neq b$, where $G(a, b)$ and $Q(a, b)$ are the classical geometric and quadratic means, $V(a, b), U(a, b), N_{Q G}(a, b)$ and $N_{G Q}(a, b)$ are Yang and Neuman mean of the second kind.

Keywords: geometric mean, quadratic mean, Neuman means of the second kind, Yang means, inequalities.

\section{Introduction}

For $a, b>0$ with $a \neq b$, the Schwab-Borchardt mean $S B(a, b)[1,2]$ is defined by

$$
S B(a, b)= \begin{cases}\frac{\sqrt{b^{2}-a^{2}}}{\cos ^{-1}(a / b)}, & \text { if } a<b, \\ \frac{\sqrt{a^{2}-b^{2}}}{\cosh ^{-1}(a / b)}, & \text { if } a>b .\end{cases}
$$

where $\cos ^{-1}(x)$ and $\cosh ^{-1}(x)=\log \left(x+\sqrt{x^{2}-1}\right)$ are the inverse cosine and inverse hyperbolic cosine functions, respectively.

It is well-known that $S B(a, b)$ is strictly increasing in both $a$ and $b$, nonsymmetric and homogeneous of degree 1 with respect to $a$ and $b$. Many symmetric bivariate means are special cases of the SchwabBorchardt mean, for example, the first and second Seiffert means, Neuman-Sándor mean, logarithmic mean and two Yang means [3] are respectively defined by

$$
\begin{aligned}
& P=P(a, b)=\frac{a-b}{2 \sin ^{-1}[(a-b) /(a+b)]}=S B(G, A), \\
& T=T(a, b)=\frac{a-b}{2 \tan ^{-1}[(a-b) /(a+b)]}=S B(A, Q),
\end{aligned}
$$




$$
\begin{gathered}
M=M(a, b)=\frac{a-b}{2 \sinh ^{-1}[(a-b) /(a+b)]}=S B(Q, A), \\
L=L(a, b)=\frac{a-b}{2 \tanh ^{-1}[(a-b) /(a+b)]}=S B(A, G), \\
U=U(a, b)=\frac{a-b}{\sqrt{2} \tan ^{-1}[(a-b) / \sqrt{2 a b}]}=S B(G, Q),
\end{gathered}
$$

and

$$
V=V(a, b)=\frac{a-b}{\sqrt{2} \sinh ^{-1}[(a-b) / \sqrt{2 a b}]}=S B(Q, G) .
$$

where $G=G(a, b)=\sqrt{a b}, A=A(a, b)=(a+b) / 2$ and $Q=Q(a, b)=\sqrt{\left(a^{2}+b^{2}\right) / 2}$ are the classical geometric, arithmetic and quadratic means of $a$ and $b$.

Let $X=X(a, b)$ and $Y=Y(a, b)$ be the symmetric bivariate means of $a$ and $b$. Then Neuman mean of the second kind $N_{X Y}(a, b)[4]$ is defined by

$$
N_{X Y}(a, b)=\frac{1}{2}\left[X+\frac{Y^{2}}{S B(X, Y)}\right] .
$$

Moreover, without loss of generality, let $a>b, v=(a-b) /(a+b) \in(0,1)$, then Neuman [4] gave explicit formulas

$$
\begin{aligned}
& N_{A G}(a, b)=\frac{1}{2} A\left[1+\left(1-v^{2}\right) \frac{\tanh ^{-1}(v)}{v}\right], N_{G A}(a, b)=\frac{1}{2} A\left[\sqrt{1-v^{2}}+\frac{\sin ^{-1}(v)}{v}\right] \\
& N_{A Q}(a, b)=\frac{1}{2} A\left[1+\left(1+v^{2}\right) \frac{\tan ^{-1}(v)}{v}\right], N_{Q A}(a, b)=\frac{1}{2} A\left[\sqrt{1+v^{2}}+\frac{\sinh ^{-1}(v)}{v}\right]
\end{aligned}
$$

and inequalities

$$
\begin{gathered}
G(a, b)<L(a, b)<N_{A G}(a, b)<P(a, b)<N_{G A}(a, b)<A(a, b) \\
<M(a, b)<N_{Q A}(a, b)<T(a, b)<N_{A Q}(a, b)<Q(a, b) .
\end{gathered}
$$

for all $a, b>0$ with $a \neq b$.

In the recent past, the Schwab-Borchardt mean has been the subject of intensive research. In particular, many remarkable inequalities for Schwab-Borchardt mean and its generated means can be found in the literature [4-14].

In [4], Neuman found the best possible constants $\alpha_{1}, \alpha_{2}, \alpha_{3}, \alpha_{4}$ and $\beta_{1}, \beta_{2}, \beta_{3}, \beta_{4}$ such that the double inequalities

$$
\begin{aligned}
& \alpha_{1} A(a, b)+\left(1-\alpha_{1}\right) G(a, b)<N_{G A}(a, b)<\beta_{1} A(a, b)+\left(1-\beta_{1}\right) G(a, b) \\
& \alpha_{2} Q(a, b)+\left(1-\alpha_{2}\right) A(a, b)<N_{A Q}(a, b)<\beta_{2} Q(a, b)+\left(1-\beta_{2}\right) A(a, b) \\
& \alpha_{3} A(a, b)+\left(1-\alpha_{3}\right) G(a, b)<N_{A G}(a, b)<\beta_{3} A(a, b)+\left(1-\beta_{3}\right) G(a, b) \\
& \alpha_{4} Q(a, b)+\left(1-\alpha_{4}\right) A(a, b)<N_{Q A}(a, b)<\beta_{4} Q(a, b)+\left(1-\beta_{4}\right) A(a, b)
\end{aligned}
$$

hold for $a, b>0$ with $a \neq b$ if and only if $\alpha_{1} \leq 2 / 3, \beta_{1} \geq \pi / 4, \alpha_{2} \leq 2 / 3, \beta_{2} \geq(\pi-2) /[4(\sqrt{2}-1)]=$ $0.689 \cdots, \alpha_{3} \leq 1 / 3, \beta_{3} \geq 1 / 2$ and $\alpha_{4} \leq 1 / 3, \beta_{4} \geq[\log (1+\sqrt{2})+\sqrt{2}-2] /[2(\sqrt{2}-1)]=0.356 \cdots$

Zhang et al. [11] presented the best possible parameters $\alpha_{1}, \alpha_{2}, \beta_{1}, \beta_{2} \in[0,1 / 2]$ and $\alpha_{3}, \alpha_{4}, \beta_{3}, \beta_{4} \in$ $[1 / 2,1]$ such that the double inequalities

$$
\begin{aligned}
& G\left(\alpha_{1} a+\left(1-\alpha_{1}\right) b, \alpha_{1} b+\left(1-\alpha_{1}\right) a\right)<N_{A G}(a, b)<G\left(\beta_{1} a+\left(1-\beta_{1}\right) b, \beta_{1} b+\left(1-\beta_{1}\right) a\right) \\
& G\left(\alpha_{2} a+\left(1-\alpha_{2}\right) b, \alpha_{2} b+\left(1-\alpha_{2}\right) a\right)<N_{G A}(a, b)<G\left(\beta_{2} a+\left(1-\beta_{2}\right) b, \beta_{2} b+\left(1-\beta_{2}\right) a\right) \\
& Q\left(\alpha_{3} a+\left(1-\alpha_{3}\right) b, \alpha_{3} b+\left(1-\alpha_{3}\right) a\right)<N_{Q A}(a, b)<Q\left(\beta_{3} a+\left(1-\beta_{3}\right) b, \beta_{3} b+\left(1-\beta_{3}\right) a\right) \\
& Q\left(\alpha_{4} a+\left(1-\alpha_{4}\right) b, \alpha_{4} b+\left(1-\alpha_{4}\right) a\right)<N_{A Q}(a, b)<Q\left(\beta_{4} a+\left(1-\beta_{4}\right) b, \beta_{4} b+\left(1-\beta_{4}\right) a\right) .
\end{aligned}
$$


hold for all $a, b>0$ with $a \neq b$.

Guo et.al. [12] proved that the double inequalities

$$
\begin{aligned}
& A^{p_{1}}(a, b) G^{1-p_{1}}(a, b)<N_{G A}(a, b)<A^{q_{1}}(a, b) G^{1-q_{1}}(a, b), \\
& \frac{p_{2}}{G(a, b)}+\frac{1-p_{2}}{A(a, b)}<N_{G A}(a, b)<\frac{q_{2}}{G(a, b)}+\frac{1-q_{2}}{A(a, b)}, \\
& A^{p_{3}}(a, b) G^{1-p_{3}}(a, b)<N_{A G}(a, b)<A^{q_{3}}(a, b) G^{1-q_{3}}(a, b), \\
& \frac{p_{4}}{G(a, b)}+\frac{1-p_{4}}{A(a, b)}<N_{A G}(a, b)<\frac{q_{4}}{G(a, b)}+\frac{1-q_{4}}{A(a, b)}, \\
& Q^{p_{5}}(a, b) A^{1-p_{5}}(a, b)<N_{A Q}(a, b)<Q^{q_{5}}(a, b) A^{1-q_{5}}(a, b), \\
& \frac{p_{6}}{A(a, b)}+\frac{1-p_{6}}{Q(a, b)}<N_{A Q}(a, b)<\frac{q_{6}}{A(a, b)}+\frac{1-q_{6}}{Q(a, b)}, \\
& Q^{p_{7}}(a, b) A^{1-p_{7}}(a, b)<N_{Q A}(a, b)<Q^{q_{7}}(a, b) A^{1-q_{7}}(a, b), \\
& \frac{p_{8}}{A(a, b)}+\frac{1-p_{8}}{Q(a, b)}<N_{Q A}(a, b)<\frac{q_{8}}{A(a, b)}+\frac{1-q_{8}}{Q(a, b)} .
\end{aligned}
$$

hold for all $a, b>0$ with $a \neq b$ if and only if $p_{1} \leq 2 / 3, q_{1} \geq 1, p_{2} \leq 0, q_{2} \geq 1 / 3, p_{3} \leq 1 / 3, q_{3} \geq 1, p_{4} \leq 0$, $q_{4} \geq 2 / 3, p_{5} \leq 2 / 3, q_{5} \geq 2 \log (\pi+2) / \log 2-4=0.7244 \cdots, p_{6} \leq[6+2 \sqrt{2}-(1+\sqrt{2}) \pi] /(\pi+2)=0.2419 \cdots$, $q_{6} \geq 1 / 3, p_{7} \leq 1 / 3, q_{7} \geq 2 \log [\sqrt{2}+\log (1+\sqrt{2})] / \log 2-2=0.3977 \cdots$ and $p_{8} \leq[2+\sqrt{2}-(1+\sqrt{2}) \log (1+$ $\sqrt{2})] /[\sqrt{2}+\log (1+\sqrt{2})]=0.5603 \cdots, q_{8} \geq 2 / 3$.

Let $a>b>0, u=(a-b) / \sqrt{2 a b} \in(0,+\infty)$. Then from (1)-(3) we gave the explicit formulas

$$
\begin{aligned}
& N_{Q G}(a, b)=\frac{1}{2} G(a, b)\left[\sqrt{1+u^{2}}+\frac{\sinh ^{-1}(u)}{u}\right] . \\
& N_{G Q}(a, b)=\frac{1}{2} G(a, b)\left[1+\left(1+u^{2}\right) \frac{\tan ^{-1}(u)}{u}\right] .
\end{aligned}
$$

The main purpose of this paper is to find the best possible parameters $\alpha_{i}, \beta_{i} \in(0,1)(i=1,2,3, \cdots, 6)$ such that the double inequalities

$$
\begin{aligned}
\alpha_{1} Q(a, b)+\left(1-\alpha_{1}\right) G(a, b) & <N_{Q G}(a, b)<\beta_{1} Q(a, b)+\left(1-\beta_{1}\right) G(a, b), \\
\frac{\alpha_{2}}{G(a, b)}+\frac{1-\alpha_{2}}{Q(a, b)} & <\frac{1}{N_{Q G}(a, b)}<\frac{\beta_{2}}{G(a, b)}+\frac{1-\beta_{2}}{Q(a, b)}, \\
\alpha_{3} Q(a, b)+\left(1-\alpha_{3}\right) G(a, b) & <N_{G Q}(a, b)<\beta_{3} Q(a, b)+\left(1-\beta_{3}\right) G(a, b), \\
\frac{\alpha_{4}}{G(a, b)}+\frac{1-\alpha_{4}}{Q(a, b)} & <\frac{1}{N_{G Q}(a, b)}<\frac{\beta_{4}}{G(a, b)}+\frac{1-\beta_{4}}{Q(a, b)}, \\
\alpha_{5} Q(a, b)+\left(1-\alpha_{5}\right) V(a, b) & <N_{Q G}(a, b)<\beta_{5} Q(a, b)+\left(1-\beta_{5}\right) V(a, b), \\
\alpha_{6} Q(a, b)+\left(1-\alpha_{6}\right) U(a, b) & <N_{G Q}(a, b)<\beta_{6} Q(a, b)+\left(1-\beta_{6}\right) U(a, b) .
\end{aligned}
$$

hold for all $a, b>0$ with $a \neq b$.

\section{Lemma}

In order to prove our main results we need several lemmas, which we present in this section.

Lemma 2.1 (see[15]) For $-\infty<a<b<+\infty$, let $f, g:[a, b] \rightarrow R$ be continuous on $[a, b]$, and be differentiable on $(a, b)$, let $g^{\prime}(x) \neq 0$ on $(a, b)$. If $f^{\prime}(x) / g^{\prime}(x)$ is increasing (decreasing) on $(a, b)$, then so are

$$
\frac{f(x)-f(a)}{g(x)-g(a)}, \frac{f(x)-f(b)}{g(x)-g(b)}
$$

If $f^{\prime}(x) / g^{\prime}(x)$ is strictly monotone, then the monotonicity in the conclusion is also strict. 
Lemma 2.2 (see [16]). Suppose that the power series $f(x)=\sum_{n=0}^{\infty} a_{n} x^{n}$ and $g(x)=\sum_{n=0}^{\infty} b_{n} x^{n}$ have the radius of convergence $r>0$ with $a_{n}, b_{n}>0$ for all $n=0,1,2, \cdots$. Let $h(x)=f(x) / g(x)$, if the sequence series $\left\{a_{n} / b_{n}\right\}_{n=0}^{\infty}$ is (strictly) increasing (decreasing), then $h(x)$ is also (strictly) increasing (decreasing) on $(0, r)$.

Lemma 2.3 (1) (See [17], Lemma 2.4) The function

$$
\varphi_{1}(x)=\frac{2 x+\sinh (2 x)-4 \sinh (x)}{\sinh (2 x)-2 \sinh (x)}
$$

is strictly increasing from $(0,+\infty)$ onto $(2 / 3,1)$.

(2)(See [17], Lemma 2.6) The function

$$
\varphi_{2}(x)=\frac{\sinh (x) \cosh (x)-x}{[\cosh (x)-1][x+\sinh (x) \cosh (x)]}
$$

is strictly decreasing from $(0,+\infty)$ onto $(0,2 / 3)$.

(3) (See [17], Lemma 2.5) The function

$$
\varphi_{3}(x)=\frac{2 x-\sin (2 x)}{\sin (x)[1-\cos (x)]}
$$

is strictly increasing from $(0, \pi / 2)$ onto $(8 / 3, \pi)$.

(4)(See [17], Lemma 2.8) The function

$$
\varphi_{4}(x)=\frac{\sin (x) \cos (x)-x}{[1-\cos (x)][x+\sin (x) \cos (x)]}
$$

is strictly decreasing from $(0, \pi / 2)$ onto $(-1,-2 / 3)$.

Lemma 2.4 The function

$$
\varphi_{5}(x)=\frac{x \sinh (2 x)-2 x^{2}}{x \sinh (2 x)-\cosh (2 x)+1}
$$

is strictly decreasing from $(0,+\infty)$ onto $(1,2)$.

Proof. Let $f_{1}(x)=x \sinh (2 x)-2 x^{2}, g_{1}(x)=x \sinh (2 x)-\cosh (2 x)+1$. Then simple computations lead to

$$
\begin{gathered}
\varphi_{5}(x)=\frac{f_{1}(x)}{g_{1}(x)}=\frac{f_{1}(x)-f_{1}\left(0^{+}\right)}{g_{1}(x)-g_{1}\left(0^{+}\right)} . \\
\frac{f_{1}^{\prime}(x)}{g_{1}^{\prime}(x)}=\frac{\sinh (2 x)+2 x \cosh (2 x)-4 x}{2 x \cosh (2 x)-\sinh (2 x)} \\
=\frac{2 x \sum_{n=0}^{\infty} \frac{2^{2 n}}{(2 n) !} x^{2 n}+\sum_{n=0}^{\infty} \frac{2^{2 n+1}}{(2 n+1) !} x^{2 n+1}-4 x}{2 x \sum_{n=0}^{\infty} \frac{2^{2 n}}{(2 n) !} x^{2 n}-\sum_{n=0}^{\infty} \frac{2^{2 n+1}}{(2 n+1) !} x^{2 n+1}} \\
=\frac{\sum_{n=1}^{\infty} \frac{(n+1) \times 2^{2 n+2}}{(2 n+1) !} x^{2 n+1}}{\sum_{n=1}^{\infty} \frac{n \times 2^{2 n+2}}{(2 n+1) !} x^{2 n+1}}=\frac{\sum_{n=0}^{\infty} \frac{(n+2) \times 2^{2 n+4}}{(2 n+3) !} x^{2 n}}{\sum_{n=0}^{\infty} \frac{(n+1) \times 2^{2 n+4}}{(2 n+3) !} x^{2 n}} .
\end{gathered}
$$

Let

$$
a_{n}=\frac{(n+2) \times 2^{2 n+4}}{(2 n+3) !}>0, b_{n}=\frac{(n+1) \times 2^{2 n+4}}{(2 n+3) !}>0 .
$$

and

$$
\frac{a_{n+1}}{b_{n+1}}-\frac{a_{n}}{b_{n}}=-\frac{1}{(n+1)(n+2)}<0 .
$$


for all $n \geq 0$.

It follows from Lemma 2.2 and (7)-(9) that $f_{1}^{\prime}(x) / g_{1}^{\prime}(x)$ is strictly decreasing on $(0,+\infty)$. Note that

$$
\varphi_{5}\left(0^{+}\right)=\frac{a_{0}}{b_{0}}=2, \varphi_{5}(+\infty)=1 .
$$

Therefore, Lemma 2.4 follows easily from Lemma 2.1 and (6), (10) together with the monotonicity of $f_{1}^{\prime}(x) / g_{1}^{\prime}(x)$.

Lemma 2.5 The function

$$
\varphi_{6}(x)=\frac{x^{2}+x \sin (x) \cos (x)-2 \sin ^{2}(x)}{\sin (x)[x-\sin (x)]}
$$

is strictly increasing from $(0, \pi / 2)$ onto $\left(0,\left(\pi^{2}-8\right) /[2(\pi-2)]\right)$.

Proof.The function $\varphi_{6}(x)$ can be rewritten as

$$
\varphi_{6}(x)=\frac{x}{\sin (x)}+\frac{x+x \cos (x)-2 \sin (x)}{x-\sin (x)}=\varphi_{7}(x)+\varphi_{8}(x) .
$$

where $\varphi_{7}(x)=x / \sin (x)$ and $\varphi_{8}(x)=[x+x \cos (x)-2 \sin (x)] /[x-\sin (x)]$.

Let $f_{2}(x)=x+x \cos (x)-2 \sin (x), g_{2}(x)=x-\sin (x), f_{3}(x)=1-\cos (x)-x \sin (x)$ and $g_{3}(x)=1-\cos (x)$. Then simple computations lead to

$$
\begin{aligned}
& \varphi_{8}(x)=\frac{f_{2}(x)}{g_{2}(x)}=\frac{f_{2}(x)-f_{2}\left(0^{+}\right)}{g_{2}(x)-g_{2}\left(0^{+}\right)} . \\
& \frac{f_{2}^{\prime}(x)}{g_{2}^{\prime}(x)}=\frac{f_{3}(x)}{g_{3}(x)}=\frac{f_{3}(x)-f_{3}\left(0^{+}\right)}{g_{3}(x)-g_{3}\left(0^{+}\right)} .
\end{aligned}
$$

and

$$
\frac{f_{3}^{\prime}(x)}{g_{3}^{\prime}(x)}=-\frac{x}{\tan (x)} .
$$

Since the function $x \rightarrow x / \tan (x)$ is strictly decreasing on $(0, \pi / 2)$, hence Lemma 2.1 and (12)-(14) lead to that $\varphi_{8}(x)$ is strictly increasing on $(0, \pi / 2)$. From (11) and the fact that the function $\varphi_{7}(x)=x / \sin (x)$ is strictly increasing on $(0, \pi / 2)$ together with the monotonicity of $\varphi_{8}(x)$ we can reach the conclusion that $\varphi_{6}(x)$ is strictly increasing on $(0, \pi / 2)$.

Note that

$$
\varphi_{6}\left(0^{+}\right)=0, \varphi_{6}\left(\frac{\pi}{2}\right)=\frac{\pi^{2}-8}{2(\pi-2)} .
$$

Therefore, Lemma 2.5 follows easily from (15) and the monotonicity of $\varphi_{6}(x)$.

\section{Main Results}

Theorem 3.1 The double inequalities

$$
\begin{gathered}
\alpha_{1} Q(a, b)+\left(1-\alpha_{1}\right) G(a, b)<N_{Q G}(a, b)<\beta_{1} Q(a, b)+\left(1-\beta_{1}\right) G(a, b) . \\
\frac{\alpha_{2}}{G(a, b)}+\frac{1-\alpha_{2}}{Q(a, b)}<\frac{1}{N_{Q G}(a, b)}<\frac{\beta_{2}}{G(a, b)}+\frac{1-\beta_{2}}{Q(a, b)} .
\end{gathered}
$$

hold for all $a, b>0$ with $a \neq b$ if and only if $\alpha_{1} \leq 1 / 3, \beta_{1} \geq 1 / 2, \alpha_{2} \leq 0$ and $\beta_{2} \geq 2 / 3$.

Proof.We clearly see that inequalities (16) and (17) can be rewritten as

$$
\alpha_{1}<\frac{N_{Q G}(a, b)-G(a, b)}{Q(a, b)-G(a, b)}<\beta_{1} .
$$

and

$$
\alpha_{2}<\frac{1 / N_{Q G}(a, b)-1 / Q(a, b)}{1 / G(a, b)-1 / Q(a, b)}<\beta_{2} .
$$


respectively.

Since both the geometric mean $G(a, b)$ and quadratic mean $Q(a, b)$ are symmetric and homogeneous of degree 1 , without loss of generality, we assume that $a>b>0$. Let $u=(a-b) / \sqrt{2 a b} \in(0,+\infty)$. Then from (4) and (18)-(19) together with $Q(a, b)=G(a, b) \sqrt{1+u^{2}}$ we have

$$
\alpha_{1}<\frac{\frac{1}{2}\left[\sqrt{1+u^{2}}+\frac{\sinh ^{-1}(u)}{u}\right]-1}{\sqrt{1+u^{2}}-1}<\beta_{1} .
$$

and

$$
\alpha_{2}<\frac{u \sqrt{1+u^{2}}-\sinh ^{-1}(u)}{\left(\sqrt{1+u^{2}}-1\right)\left[u \sqrt{1+u^{2}}+\sinh ^{-1}(u)\right]}<\beta_{2} .
$$

respectively

Let $x=\sinh ^{-1}(u)$. Then $x \in(0,+\infty)$,

$$
\begin{aligned}
& \frac{\frac{1}{2}\left[\sqrt{1+u^{2}}+\frac{\sinh ^{-1}(u)}{u}\right]-1}{\sqrt{1+u^{2}}-1} \\
&= \frac{1}{2} \frac{2 x+\sinh (2 x)-4 \sinh (x)}{\sinh (2 x)-2 \sinh (x)}=\frac{1}{2} \varphi_{1}(x) . \\
& \frac{u \sqrt{1+u^{2}}-\sinh ^{-1}(u)}{\left(\sqrt{1+u^{2}}-1\right)\left[u \sqrt{1+u^{2}}+\sinh ^{-1}(u)\right]} \\
&=\frac{\sinh (x) \cosh (x)-x}{[\cosh (x)-1][x+\sinh (x) \cosh (x)]}=\varphi_{2}(x) .
\end{aligned}
$$

where the functions $\varphi_{1}(x)$ and $\varphi_{2}(x)$ are defined as in Lemma 2.3(1) and (2).

Therefore, inequality (16) holds for all $a, b>0$ with $a \neq b$ if and only if $\alpha_{1} \leq 1 / 3$ and $\beta_{1} \geq 1 / 2$ follows from (20) and (22) together with Lemma 2.3(1), inequality (17) holds for all $a, b>0$ with $a \neq b$ if and only if $\alpha_{2} \leq 0$ and $\beta_{2} \geq 2 / 3$ follows from (21) and (23) together with Lemma 2.3(2).

Theorem 3.2 The double inequalities

$$
\begin{gathered}
\alpha_{3} Q(a, b)+\left(1-\alpha_{3}\right) G(a, b)<N_{G Q}(a, b)<\beta_{3} Q(a, b)+\left(1-\beta_{3}\right) G(a, b) . \\
\frac{\alpha_{4}}{G(a, b)}+\frac{1-\alpha_{4}}{Q(a, b)}<\frac{1}{N_{G Q}(a, b)}<\frac{\beta_{4}}{G(a, b)}+\frac{1-\beta_{4}}{Q(a, b)} .
\end{gathered}
$$

holds for all $a, b>0$ with $a \neq b$ if and only if $\alpha_{3} \leq 2 / 3, \beta_{3} \geq \pi / 4, \alpha_{4} \leq 0$ and $\beta_{4} \geq 1 / 3$.

Proof.We clearly see that inequalities (24) and (25) can be rewritten as

$$
\alpha_{3}<\frac{N_{G Q}(a, b)-G(a, b)}{Q(a, b)-G(a, b)}<\beta_{3} .
$$

and

$$
\alpha_{4}<\frac{1 / N_{G Q}(a, b)-1 / Q(a, b)}{1 / G(a, b)-1 / Q(a, b)}<\beta_{4} .
$$

respectively.

Since both the geometric mean $G(a, b)$ and quadratic mean $Q(a, b)$ are symmetric and homogeneous of degree 1 , without loss of generality, we assume that $a>b>0$. Let $u=(a-b) / \sqrt{2 a b} \in(0,+\infty)$. Then from (5) and (26)-(27) together with $Q(a, b)=G(a, b) \sqrt{1+u^{2}}$ we have

$$
\alpha_{3}<\frac{\frac{1}{2}\left[1+\left(1+u^{2}\right) \frac{\tan ^{-1}(u)}{u}\right]-1}{\sqrt{1+u^{2}}-1}<\beta_{3} .
$$


and

$$
\alpha_{4}<\frac{2 u \sqrt{1+u^{2}}-\left[u+\left(1+u^{2}\right) \tan ^{-1}(u)\right]}{\left(\sqrt{1+u^{2}}-1\right)\left[u+\left(1+u^{2}\right) \tan ^{-1}(u)\right]}<\beta_{4} .
$$

respectively.

Let $x=\tan ^{-1}(u)$. Then $x \in(0, \pi / 2)$,

$$
\begin{gathered}
\frac{\frac{1}{2}\left[1+\left(1+u^{2}\right) \frac{\tan ^{-1}(u)}{u}\right]-1}{\sqrt{1+u^{2}}-1} \\
=\frac{1}{4} \frac{2 x-\sin (2 x)}{\sin (x)[1-\cos (x)]}=\frac{1}{4} \varphi_{3}(x) . \\
\frac{2 u \sqrt{1+u^{2}}-\left[u+\left(1+u^{2}\right) \tan ^{-1}(u)\right]}{\left(\sqrt{1+u^{2}}-1\right)\left[u+\left(1+u^{2}\right) \tan ^{-1}(u)\right]} \\
=1+\frac{\sin (x) \cos (x)-x}{[1-\cos (x)][x+\sin (x) \cos (x)]}=1+\varphi_{4}(x) .
\end{gathered}
$$

where the functions $\varphi_{3}(x)$ and $\varphi_{4}(x)$ are defined as in Lemma 2.3(3) and 2.3(4).

Therefore, inequality (24) holds for all $a, b>0$ with $a \neq b$ if and only if $\alpha_{3} \leq 2 / 3$ and $\beta_{3} \geq \pi / 4$ follows from (28) and (30) together with Lemma 2.3(3), inequality (25) holds for all $a, b>0$ with $a \neq b$ if and only if $\alpha_{4} \leq 0$ and $\beta_{4} \geq 1 / 3$ follows from (29) and (31) together with Lemma 2.3(4).

Theorem 3.3 The double inequalities

$$
\alpha_{5} Q(a, b)+\left(1-\alpha_{5}\right) V(a, b)<N_{Q G}(a, b)<\beta_{5} Q(a, b)+\left(1-\beta_{5}\right) V(a, b) .
$$

holds for all $a, b>0$ with $a \neq b$ if and only if $\alpha_{5} \leq 0$ and $\beta_{5} \geq 1 / 2$.

Proof. We clearly see that inequalities (32) can be rewritten as

$$
\alpha_{5}<\frac{N_{Q G}(a, b)-V(a, b)}{Q(a, b)-V(a, b)}<\beta_{5} .
$$

Since both the geometric mean $G(a, b)$ and quadratic mean $Q(a, b)$ are symmetric and homogeneous of degree 1 , without loss of generality, we assume that $a>b>0$. Let $u=(a-b) / \sqrt{2 a b} \in(0,+\infty)$. Then from (4) and (33) together with $Q(a, b)=G(a, b) \sqrt{1+u^{2}}$ we have

$$
\alpha_{5}<\frac{\frac{1}{2}\left[\sqrt{1+u^{2}}+\frac{\sinh ^{-1}(u)}{u}\right]-\frac{u}{\sinh ^{-1}(u)}}{\sqrt{1+u^{2}}-\frac{u}{\sinh ^{-1}(u)}}<\beta_{5} .
$$

Let $x=\sinh ^{-1}(u)$. Then $x \in(0,+\infty)$,

$$
\begin{aligned}
& \frac{\frac{1}{2}\left[\sqrt{1+u^{2}}+\frac{\sinh ^{-1}(u)}{u}\right]-\frac{u}{\sinh ^{-1}(u)}}{\sqrt{1+u^{2}}-\frac{u}{\sinh ^{-1}(u)}} \\
& =1-\frac{1}{2} \frac{x \sinh (2 x)-2 x^{2}}{x \sinh (2 x)-\cosh (2 x)+1}=1-\frac{1}{2} \varphi_{5}(x) .
\end{aligned}
$$

where the functions $\varphi_{5}(x)$ is defined as in Lemma 2.4.

Therefore, inequality (32) holds for all $a, b>0$ with $a \neq b$ if and only if $\alpha_{5} \leq 0$ and $\beta_{5} \geq 1 / 2$ follows from (34) and (35) together with Lemma 2.4. 
Theorem 3.4 The double inequalities

$$
\alpha_{6} Q(a, b)+\left(1-\alpha_{6}\right) U(a, b)<N_{G Q}(a, b)<\beta_{6} Q(a, b)+\left(1-\beta_{6}\right) U(a, b) .
$$

holds for all $a, b>0$ with $a \neq b$ if and only if $\alpha_{6} \leq 0, \beta_{6} \geq\left(\pi^{2}-8\right) /[4(\pi-2)]=0.4094 \cdots$.

Proof.We clearly see that inequalities (36) can be rewritten as

$$
\alpha_{6}<\frac{N_{G Q}(a, b)-U(a, b)}{Q(a, b)-U(a, b)}<\beta_{6}
$$

Since both the geometric mean $G(a, b)$ and quadratic mean $Q(a, b)$ are symmetric and homogeneous of degree 1 , without loss of generality, we assume that $a>b>0$. Let $u=(a-b) / \sqrt{2 a b} \in(0,+\infty)$. Then from (5) and (36) together with $Q(a, b)=G(a, b) \sqrt{1+u^{2}}$ we have

$$
\alpha_{6}<\frac{\frac{1}{2}\left[1+\left(1+u^{2}\right) \frac{\tan ^{-1}(u)}{u}\right]-\frac{u}{\tan ^{-1}(u)}}{\sqrt{1+u^{2}}-\frac{u}{\tan ^{-1}(u)}}<\beta_{6} .
$$

Let $x=\tan ^{-1}(u)$. Then $x \in(0, \pi / 2)$,

$$
\frac{\frac{1}{2}\left[1+\left(1+u^{2}\right) \frac{\tan ^{-1}(u)}{u}\right]-\frac{u}{\tan ^{-1}(u)}}{\sqrt{1+u^{2}}-\frac{u}{\tan ^{-1}(u)}}=\frac{1}{2} \varphi_{6}(x),
$$

where the function $\varphi_{6}(x)$ is defined as in Lemma 2.5 .

Therefore, inequality (36) holds for all $a, b>0$ with $a \neq b$ if and only if $\alpha_{6} \leq 0$ and $\beta_{6} \geq\left(\pi^{2}-\right.$ $8) /[4(\pi-2)]=0.4094 \cdots$ follows from $(37)-(39)$ together with Lemma 2.5 .

\section{Applications}

In this section, we will establish several sharp inequalities involving the hyperbolic, inverse hyperbolic, trigonometric and inverse trigonometric functions by use of Theorems 3.1-3.4.

From (3) we clearly see that

$$
N_{Q G}(a, b)=\frac{1}{2}\left[Q(a, b)+\frac{G^{2}(a, b)}{V(a, b)}\right], N_{G Q}(a, b)=\frac{1}{2}\left[G(a, b)+\frac{Q^{2}(a, b)}{U(a, b)}\right] .
$$

Let $a>b$ and $x=\sinh ^{-1}\left(\frac{a-b}{\sqrt{2 a b}}\right) \in(0, \infty)$. Then simple computations lead to

$$
\frac{Q(a, b)}{G(a, b)}=\cosh (x), \frac{V(a, b)}{G(a, b)}=\frac{\sinh (x)}{x}, \frac{U(a, b)}{G(a, b)}=\frac{\sinh (x)}{\tan ^{-1}[\sinh (x)]} .
$$

Theorems 3.1-3.4 and (40)-(41) lead to Theorem 4.1.

Theorem 4.1 The double inequalities

$$
\begin{gathered}
2 \alpha_{1} \cosh (x)+2\left(1-\alpha_{1}\right)<\cosh (x)+\frac{x}{\sinh (x)}<2 \beta_{1} \cosh (x)+2\left(1-\beta_{1}\right), \\
\frac{1}{2}\left[\alpha_{2} \cosh (x)+\left(1-\alpha_{2}\right)\right]<1-\frac{2 x}{\sinh (2 x)+2 x}<\frac{1}{2}\left[\beta_{2} \cosh (x)+\left(1-\beta_{2}\right)\right], \\
2 \alpha_{3} \cosh (x)+\left(1-2 \alpha_{3}\right)<\cosh (x) \operatorname{coth}(x) \tan ^{-1}[\sinh (x)]<2 \beta_{3} \cosh (x)+\left(1-2 \beta_{3}\right), \\
\frac{\alpha_{4} \cosh (x)+\left(1-\alpha_{4}\right)}{2 \cosh (x)}<\frac{1}{1+\cosh (x) \operatorname{coth}(x) \tan ^{-1}[\sinh (x)]}<\frac{\beta_{4} \cosh (x)+\left(1-\beta_{4}\right)}{2 \cosh (x)},
\end{gathered}
$$




$$
\begin{gathered}
2 \alpha_{5} \cosh (x)+2\left(1-\alpha_{5}\right) \frac{\sinh (x)}{x}<\cosh (x)+\frac{x}{\sinh (x)}<2 \beta_{5} \cosh (x)+2\left(1-\beta_{5}\right) \frac{\sinh (x)}{x} \\
2 \alpha_{6} \cosh (x)+2\left(1-\alpha_{6}\right) \frac{\sinh (x)}{\tan ^{-1}[\sinh (x)]}<1+\cosh (x) \operatorname{coth}(x) \tan ^{-1}[\sinh (x)] \\
<2 \beta_{6} \cosh (x)+2\left(1-\beta_{6}\right) \frac{\sinh (x)}{\tan ^{-1}[\sinh (x)]} .
\end{gathered}
$$

hold for all $x>0$ if and only if $\alpha_{1} \leq 1 / 3, \beta_{1} \geq 1 / 2, \alpha_{2} \leq 0, \beta_{2} \geq 2 / 3, \alpha_{3} \leq 2 / 3, \beta_{3} \geq \pi / 4, \alpha_{4} \leq 0$, $\beta_{4} \geq 1 / 3, \alpha_{5} \leq 0, \beta_{5} \geq 1 / 2, \alpha_{6} \leq 0$ and $\beta_{6} \geq\left(\pi^{2}-8\right) /[4(\pi-2)]$.

Let $a>b$ and $x=\tan ^{-1}\left(\frac{a-b}{\sqrt{2 a b}}\right) \in(0, \pi / 2)$. Then it is not difficult to verify that

$$
\frac{Q(a, b)}{G(a, b)}=\sec (x), \frac{V(a, b)}{G(a, b)}=\frac{\tan (x)}{\sinh ^{-1}[\tan (x)]}, \frac{U(a, b)}{G(a, b)}=\frac{\tan (x)}{x} .
$$

From Theorems 3.1-3.4 and (40), (42) we get Theorem 4.2 immediately.

Theorem 4.2 The double inequalities

$$
\begin{gathered}
2 \alpha_{1} \sec (x)+2\left(1-\alpha_{1}\right)<\sec (x)+\frac{\sinh ^{-1}[\tan (x)]}{\tan (x)}<2 \beta_{1} \sec (x)+2\left(1-\beta_{1}\right), \\
\frac{1}{2}\left[\alpha_{2}+\left(1-\alpha_{2}\right) \cos (x)\right]<\frac{\tan (x)}{\sec (x) \tan (x)+\sinh ^{-1}[\tan (x)]}<\frac{1}{2}\left[\beta_{2}+\left(1-\beta_{2}\right) \cos (x)\right], \\
2 \alpha_{3} \sec (x)+2\left(1-\alpha_{3}\right)<1+\frac{2 x}{\sin (2 x)}<2 \beta_{3} \sec (x)+2\left(1-\beta_{3}\right), \\
\frac{1}{2}\left[\alpha_{4}+\left(1-\alpha_{4}\right) \cos (x)\right]<1-\frac{2 x}{\sin (2 x)+2 x}<\frac{1}{2}\left[\beta_{4}+\left(1-\beta_{4}\right) \cos (x)\right], \\
2 \alpha_{5} \sec (x)+2\left(1-\alpha_{5}\right) \frac{\tan (x)}{\sinh ^{-1}[\tan (x)]}<\sec (x)+\frac{\sinh ^{-1}[\tan (x)]}{\tan (x)}<2 \beta_{5} \sec (x)+2\left(1-\beta_{5}\right) \frac{\tan (x)}{\sinh ^{-1}[\tan (x)]} \\
2 \alpha_{6} \sec (x)+2\left(1-\alpha_{6}\right) \frac{\tan (x)}{x}<1+\frac{2 x}{\sin (2 x)}<2 \beta_{6} \sec (x)+2\left(1-\beta_{6}\right) \frac{\tan (x)}{x} .
\end{gathered}
$$

hold for all $x \in(0, \pi / 2)$ if and only if $\alpha_{1} \leq 1 / 3, \beta_{1} \geq 1 / 2, \alpha_{2} \leq 0, \beta_{2} \geq 2 / 3, \alpha_{3} \leq 2 / 3, \beta_{3} \geq \pi / 4, \alpha_{4} \leq 0$, $\beta_{4} \geq 1 / 3, \alpha_{5} \leq 0, \beta_{5} \geq 1 / 2, \alpha_{6} \leq 0$ and $\beta_{6} \geq\left(\pi^{2}-8\right) /[4(\pi-2)]$.

Acknowledgments. This research was supported by the Natural Science Foundation of Zhejiang Broadcast and TV University under Grant XKT-15G17.

\section{References}

1. E. Neuman, and J. Sándor, "On the Schwab-Borchardt mean", Math. Pann., vol.14, no.2, pp.253-266, 2003.

2. E. Neuman, and J. Sándor, "On the Schwab-Borchardt mean II", Math. Pann., vol.17, no.1, pp.49-59, 2006.

3. Z.-H.Yang, "Three families of two-parameter means constructed by trigonometric functions", J. Inequal. Appl., 541, 27 pages(2013).

4. E. Neuman, "On a new bivariate mean", Aequat. Math., vol.88, no.3, pp.277-289, 2014.

5. E. Neuman, "On some means derived from the Schwab-Borchardt mean", J. Math. Inequal., vol.8, pp.1, pp.171-183, 2014.

6. E. Neuman, "On some means derived from the Schwab-Borchardt mean II", J. Math. Inequal.,vol.8, pp.2, 361-370, 2014.

7. Y.-M. Chu, W.-M. Qian, L.-M. Wu, and X.-H. Zhang, "Optimal bounds for the first and second Seiffert means in terms of geometric, arithmetic and contraharmonic means", J. Inequal. Appl.,44(2015).

8. Y.-M. Chu, and W.-M. Qian, "Refinements of bounds for Neuman means, Abstr". Appl. Anal., Article ID 254132, 8 pages (2004). 
9. W.-M. Qian, and Y.-M. Chu, "Optimal bounds for Neuman means in terms of geometric, arithmetic and quadratic means", J. Inequal. Appl., 2014:175 (2014).

10. W.-M. Qian, Z.-H Shao and Y.-M. Chu, "Sharp Inequalities Involving Neuman Means of the Second Kind", J. Math. Inequal., vol.9, no.2 pp.531-540, 2015.

11. Y. Zhang, Y.-M. Chu and Y.-L. Jiang, "Sharp geometric mean bounds for Neumam means", Abstr. Appl. Anal., Article ID949818, 6 pages, (2014).

12. Z.-J.Guo, Y. zhang, Y.- M. Chu and Y.-Q. Song, "Sharp bounds for Neuman means in terms of geometric, arithmetic and quadratic means", Available online at http:// arxiv. org/abs/1405.4384.

13. Y.-Y. Yang, and W.-M. Qian, "The optimal convex combination bounds of harmonic, arithmetic and contraharmonic means for the Neuman means", Int. Math. Forum, vol.9, no.27, pp.1295- 1307, 2014.

14. Z.-Y. He, Y.-M. Chu and M.-K. Wang, "Optimal bounds for Neuman means in terms of harmonic and contraharmonic means", J. Appl. Math., Article ID 807623, 4 pages(2013).

15. G. D. Anderson, M. K. Vamanamurthy and M. K. Vuorinen, Conformal Invariants, Inequalities, and Quasiconformal Maps, Canadian Mathematical Society Series of Monographs and Advanced Texts, John Wiley \& Sons, New York, NY, USA, 1997.

16. S. Simić and M. Vuorinen, "Landen inequalities for zero-balanced hypergeometric function", Abstr. Appl. Anal., Article ID 932061, 11 pages(2012).

17. S.- B. Chen, Z.-Y. He, Y.-M. Chu, Y.-Q. Song and X.-J. Tao, "Note on certain inequalities for Neuman means", J. Inequal. Appl., 2014:370(2014). 